\title{
A PARTITION PROPERTY OF SIMPLICES IN EUCLIDEAN SPACE
}

\author{
P. FRANKL AND V. RÖDL
}

\section{INTRODUCTION AND STATEMENT OF THE RESULTS}

Let $\mathbb{R}^{n}$ denote $n$-dimensional Euclidean space endowed with the standard metric. For $x, y \in \mathbb{R}^{n}$, their distance is denoted by $|x-y|$.

Definition 1.1 [E]. A subset $B$ of $\mathbb{R}^{d}$ is called Ramsey if for every $r \geq 2$ there exists some $n=n(r, B)$ with the following partition property. For every partition $\mathbb{R}^{n}=V_{1} \cup \cdots \cup V_{r}$, there exists some $j, 1 \leq j \leq r$, and $B_{j} \subset V_{j}$ such that $B$ is congruent to $B_{j}$.

In a series of papers, Erdös et al. [E] have investigated this property. They have shown that all Ramsey sets are spherical, that is, every Ramsey set is contained in an appropriate sphere. On the other hand, they have shown that the vertex set (and, therefore, all its subsets) of bricks ( $d$-dimensional parallelepipeds) is Ramsey.

The simplest sets that are spherical but cannot be embedded into the vertex set of a brick are the sets of obtuse triangles. In [FR1], it is shown that they are indeed Ramsey, using Ramsey's Theorem (cf. [G2]) and the Product Theorem of [E].

The aim of the present paper is twofold. First, we want to show that the vertex set of every nondegenerate simplex in any dimension is Ramsey. Second, we want to show that for both simplices and bricks, and even for their products, one can in fact choose $n(r, B)=c(B) \log r$, where $c(B)$ is an appropriate positive constant. The paper is organized as follows. In $\S 2$, super-Ramsey property is introduced. This notion is stronger than being Ramsey. It is shown that the direct product of super-Ramsey sets is super-Ramsey. In $\S 3$, it is shown that if every edge of an $n$-dimensional simplex is between $1-\varepsilon$ and $1+\varepsilon$ with $\varepsilon=\varepsilon(n)$ being a sufficiently small positive number, then it can be embedded into a brick, i.e., into the direct product of two-element sets. In $\S 4$, it is proved that given a nondegenerate simplex with edge lengths $a_{i j}, 1 \leq i<j \leq n$, and $\delta>0$, there exists some super-Ramsey simplex whose edge lengths $v_{i j}$ verify $\left|a_{i j}^{2}-v_{i j}^{2}\right|<\delta$ for all $1 \leq i<j \leq n$. In the proof of this result, an earlier result

Received by the editors August 25, 1988.

1980 Mathematics Subject Classification (1985 Revision). Primary 05B30, 51M20.

This paper was written while the authors were visiting AT\&T Bell Laboratories, Murray Hill, New Jersey. 
of the authors about the intersection patterns of partitions plays a crucial role. Finally, in $\S 5$ the main result is established. Namely, combining the results of $\S \S 2-4$, it is proved that all nondegenerate simplices are super-Ramsey. We close the paper with some related and open problems.

\section{AN EXPONENTIAL PRODUCT THEOREM}

For two sets $A \subset \mathbb{R}^{d}, B \subset \mathbb{R}^{e}$, their product $A * B \subset \mathbb{R}^{d+e}$ is defined by

$$
A * B=\{\mathbf{a} * \mathbf{b}: \mathbf{a} \in A, \mathbf{b} \in B\}
$$

where for $\mathbf{a}=\left(a_{1}, \ldots, a_{d}\right)$ and $\mathbf{b}=\left(b_{1}, \ldots, b_{e}\right)$ and $\mathbf{a} * \mathbf{b}=\left(a_{1}, \ldots, a_{d}\right.$, $\left.b_{1}, \ldots, b_{e}\right)$ is defined by concatenation.

In [E], it is shown that if $A$ and $B$ are both Ramsey, then so is $A * B$.

We need a similar statement for a substantially stronger property.

Definition 2.1. A set $A \subset \mathbb{R}^{d}$ is called super-Ramsey if there exist positive constants $c$ and $\varepsilon$ and subsets $X=X(n) \subset \mathbb{R}^{n}$ for every $n>n_{0}(A)$ with the following two properties:

(i) $|X|<c^{n}$;

(ii) $|Y|<|X| /(1+\varepsilon)^{n}$ holds for all subsets $Y \subset X$ that contain no congruent copy of $A$.

Theorem 2.2. If $A \subset \mathbb{R}^{d}$ and $B \subset \mathbb{R}^{e}$ are super-Ramsey, then so is $A * B$.

Proof. Let $X=X(n)$ be the set from Definition 2.1 for $A$ and, similarly, $Z=Z(m)$ for $B$, with $f, \delta$ the corresponding constants. Let $V$ be a subset of $X(n) * Z(m)$ that contains no congruent copy of $A * B$.

For $\mathbf{x} \in X(n)$ consider the set $V_{\mathbf{x}}=V \cap \mathbf{x} * Z(m)$. Since $\mathbf{x} * Z(m)$ is congruent to $Z(m)$, it contains at least $\left\lfloor\left|V_{\mathbf{x}}\right| /\left(|Z(m)| /(1+\delta)^{m}\right)\right\rfloor$ congruent copies of $B$.

Define for each $|B|$-subset $F$ of $Z(m)$ congruent to $B$ a set $Y(F) \subset X(n)$ by setting $\mathbf{x} \in Y(F)$ iff the corresponding $|B|$-subset $\mathbf{x} * F$ is contained in $V_{\mathbf{x}}$.

Since $V$ contains no congruent copy of $A * B, Y(F)$ contains no congruent copy of $A$. Consequently, by (ii) of Definition $2.1,|Y(F)|<|X| /(1+\varepsilon)^{n}$ holds.

Thus, we have

$$
\sum_{\mathbf{x} \in X}\left\lfloor\left|V_{\mathbf{x}}\right|(1+\delta)^{m} /|Z|\right\rfloor<\left(\begin{array}{c}
f^{m} \\
|B|
\end{array}\right)|X| /(1+\varepsilon)^{n} .
$$

On the other hand,

$$
\begin{aligned}
\sum_{\mathbf{x} \in X}\left\lfloor\left|V_{\mathbf{x}}\right|(1+\delta)^{m} /|Z|\right\rfloor> & \sum_{\mathbf{x} \in X}\left(\left|V_{\mathbf{x}}\right|(1+\delta)^{m} /|Z|-1\right) \\
& =|V|(1+\delta)^{m} /|Z|-|X|
\end{aligned}
$$

Comparing (2.1) and (2.2) we deduce

$$
|V|<|X * Z|\left(\left(\begin{array}{c}
f^{m} \\
|B|
\end{array}\right) /(1+\varepsilon)^{n}+1\right) /(1+\delta)^{m} .
$$

Clearly, the statement follows from (2.3). For example, define $k$ by $f^{|B|}=$ $(1+\varepsilon)^{k}$. Then for $n>k m,(2.3)$ yields $|V|<|X * Z| \cdot 2 /(1+\delta)^{m}$. 
To satisfy Definition 2.1 formally, for given $n$ we can consider $X\left(n^{\prime}\right) * Z\left(n^{\prime \prime}\right)$ with $n^{\prime \prime}=\lfloor n / k\rfloor, n=n^{\prime}+n^{\prime \prime} ; \tilde{c}=\max \{c, f\}, \tilde{\varepsilon}>0$ arbitrary with $(1+\tilde{\varepsilon})^{k}<$ $1+\delta$.

Corollary 2.3. All subsets of the vertex sets of bricks are super-Ramsey.

Proof. The fact that two-element sets are super-Ramsey is proved in [FW] (with $c=2, \varepsilon=0.2$ ). The rest follows from Theorem 2.2 .

\section{Almost REgUlar SIMPLICES}

Let $\mathbf{y}_{1}, \ldots, \mathbf{y}_{n}$ be the vertices of an $(n-1)$-dimensional simplex. Let $b_{i j}=$ $\left|\mathbf{y}_{i}-\mathbf{y}_{j}\right|^{2}$ be the square of the distance of the $i$ th and $j$ th vertices.

Consider the $\left(\begin{array}{l}n \\ 2\end{array}\right)$-dimensional brick with edges parallel to the axes and with respective lengths $x_{i j}, 1 \leq i<j \leq n$. Define $\mathbf{x}^{(i)}=\left(a_{j k}: 1 \leq j<k \leq n\right)$ by

$$
a_{j k}= \begin{cases}x_{j k} & \text { if } j=i \text { or } k=i, \\ 0 & \text { otherwise. }\end{cases}
$$

Lemma 3.1. Let $n \geq 11$. If $\varepsilon=\varepsilon(n)$ is a sufficiently small positive real number, then for all choices of $b_{i j}, 1 \leq i<j \leq n$, satisfying $\left|b_{i j}-1\right|<\varepsilon$, one can find positive reals $x_{i j}$ with

$$
\left|\mathbf{x}^{(i)}-\mathbf{x}^{(j)}\right|^{2}=b_{i j} \text { for all } 1 \leq i<j \leq n .
$$

Proof. Set $z_{i j}=x_{i j}^{2}$ and $z_{j i}=z_{i j}$. Then the $\left(\begin{array}{l}n \\ 2\end{array}\right)$ equations in (3.1) can be written as

$$
\sum_{l \neq j, i}\left(z_{i l}+z_{j l}\right)=b_{i j}, \quad 1 \leq i<j \leq n .
$$

This is a system of $\left(\begin{array}{l}n \\ 2\end{array}\right)$ linear equations in $\left(\begin{array}{l}n \\ 2\end{array}\right)$ variables. We claim that the matrix, say $M$, of the system is nondegenerate. There are many ways to prove this, but we choose a combinatorial method using an intersection theorem for sets. The $(0,1)$-matrix $M$ corresponds in a natural way to a family $\mathscr{F}=$ $\left\{F_{i j}: 1 \leq i<j \leq n\right\}$ of $2(n-2)$-subsets of $\left(\begin{array}{c}{[n]} \\ 2\end{array}\right)=\{(i, j): 1 \leq i<j \leq n\}$ in the following way:

$$
F_{i j}=\{(k, l): 1 \leq k<l \leq n,|\{i, j\} \cap\{k, l\}|=1\} .
$$

Then the $(i, j)$-row of $M$ is the characteristic vector of $F_{i j}$.

Now $\left|F_{i j} \cap F_{i j^{\prime}}\right|=n-2$ for $1 \leq j<j^{\prime} \leq n$, and $\left|F_{i j} \cap F_{k l}\right|=4$ for $\{i, j\} \cap\{k, l\}=\varnothing$. Thus, for $(n-6) \nmid(n-2)$, i.e., $(n-6) \nmid 4$, and, in particular, for $n \geq 11$, the conditions of the following theorem are fulfilled $(a=2 n-4, b=n-2, m=n-6)$.

Theorem (Frankl-Rosenberg [FR]). Let $a, b, m$ be integers and suppose that $F$ is a family of a-element subsets of a set $Y$ satisfying $\left|F \cap F^{\prime}\right| \equiv b(\bmod m)$ for all distinct $F, F^{\prime} \in \mathscr{F}$. If $a \neq \equiv(\bmod m)$, then the characteristic vectors of the sets $F \in \mathscr{F}$ are linearly independent (over the rationals).

Since in our case $|\mathscr{F}|=\left(\begin{array}{l}n \\ 2\end{array}\right)$, we infer that $M$ is a nonsingular matrix. 
Setting $z_{i j}=1 /(2(n-2))$ gives a solution for $b_{i j}=1,1 \leq i<j \leq n$. Thus, by continuity the statement follows.

Remark. As can be seen from the proof, the condition $n \geq 11$ can be weakened. However, the statement is not true for $n=4$.

Corollary 3.2. For every $n \geq 2$ there exists an $\varepsilon=\varepsilon(n)>0$ such that if $B \subset \mathbb{R}^{n}$ is an $(n+1)$-element point set with ||$x-\left.y\right|^{2}-1 \mid \leq \varepsilon$ for all distinct $x, y \in B$, then $B$ is super-Ramsey.

Proof. In view of Lemma 3.1, B is the subset of some brick in $\max \left\{\left(\begin{array}{c}n \\ 2\end{array}\right),\left(\begin{array}{c}11 \\ 2\end{array}\right)\right\}$ dimensions. Now apply Corollary 2.3 .

\section{SUPER-RAMSEY SIMPLICES ARE DENSE}

Lemma 4.1. For every $s \geq 2$ and for every $\varepsilon>0$ there is a super-Ramsey set $B=\left\{\mathbf{b}_{1}, \ldots, \mathbf{b}_{s}\right\} \subset R^{s-1}$ such that || $\mathbf{b}_{i}-\left.\mathbf{b}_{j}\right|^{2}-|i-j|^{2} \mid<\varepsilon$.

Proof. Let $t=t(\varepsilon)$ be a large integer and consider the points

$$
\mathbf{x}(i)=\left(x_{1}(i), \ldots, x_{2 t+s}(i)\right) \in R^{2 t+s}
$$

defined by

$$
x_{j}(i)= \begin{cases}0 & \text { if } j<i \text { or if } j>2 t+i, \\ j-i+1 & \text { if } i \leq j \leq t+i, \\ 2 t+1+i-j & \text { if } t+i<j \leq 2 t+i .\end{cases}
$$

For example, for $t=3, s=3$, we have $\mathbf{x}(2)=(0,1,2,3,4,3,2,1,0)$. Direct computation gives

$$
|| \mathbf{x}(i)-\left.\mathbf{x}(j)\right|^{2}-2 t(i-j)^{2} \mid<4 s^{4} .
$$

Let $M$ be an $s$ by $(t+2)^{s}$ matrix with entries $0,1, \ldots, t+1$ in which every sequence $\left(e_{1}, \ldots, e_{s}\right)$ with $0 \leq e_{i} \leq t+1$ for $1 \leq i \leq s$ occurs exactly once as a column. Let $\mathbf{y}(i) \in R^{(t+2)^{s}}$ be the $i$ th column of $M$.

Define $\mathbf{z}(i)=\mathbf{x}(i) * \mathbf{x}(i) * \cdots * \mathbf{x}(i) * \mathbf{y}(i) \in R^{m(2 t+s)+(t+2)^{s}}$, where $m>m_{0}(t)$ is a large integer.

Finally, let $p$ be a large integer, $p>p_{0}(m)$, and define

$$
\boldsymbol{\omega}(i)=\mathbf{z}(i) * \cdots * \mathbf{z}(i) \in R^{p\left(m(2 t+s)+(t+2)^{s}\right)},
$$

i.e., $\boldsymbol{\omega}(i)$ is the $p$ th power of $\mathbf{z}(i)$. Set $n=p\left(m(2 t+s)+(t+2)^{s}\right)$.

Computing $|\omega(i)-\omega(j)|^{2}$ for $1 \leq i<j \leq m$, we see that for $t>t(\varepsilon)$, $m>m_{0}(t), p>p_{0}(m)$ one has

$$
|| \omega(i)-\left.\omega(j)\right|^{2}-2 t m p(i-j)^{2} \mid<\varepsilon t m p .
$$

That is, $\mathbf{b}_{i}=\boldsymbol{\omega}(i) / \sqrt{2 t m p}$ will satisfy the distance requirements. But we have to show that $\{\boldsymbol{\omega}(1), \ldots, \boldsymbol{\omega}(s)\}$ is super-Ramsey.

This, fortunately, follows from Theorem 1.16 of [FR2]. Namely, each $\omega(i)$ defines a partition $\{1, \ldots, n\}=X_{0}^{(i)} \cup \cdots \cup X_{t+1}^{(i)}$, where $X_{j}^{(i)}=\{l$ : the $l$ th coordinate of $\boldsymbol{\omega}(i)$ is $j\}$. 
By the construction, $\left|X_{j}^{(i)}\right|=\left|X_{j}^{\left(i^{\prime}\right)}\right|$ holds for $i \neq i^{\prime}$.

Also, the intersection pattern of these partitions satisfies the assumptions of Theorem 1.16 of [FR2] if $p$ is sufficiently large.

Thus, to satisfy Definition 2.1 , we can take the set of all points $\left(x_{1}, \ldots, x_{n}\right) \in$ $R^{n}$ that have exactly $\left|X_{j}^{(1)}\right|$ coordinates equal to $j, 0 \leq j \leq t+1$. This is fewer than $(t+2)^{n}$ points. The above cited theorem guarantees that (ii) of Definition 2.1 is fulfilled with some positive $\varepsilon^{\prime}$.

This concludes the proof of the lemma.

Corollary 4.2. Let $A=\{\mathbf{a}(1), \ldots, \mathbf{a}(d)\} \subset R^{d-1}$ be an arbitrary point set and $\delta>0$ be arbitrary. Then there exists a super-Ramsey set $V=\{\mathbf{v}(1), \ldots, \mathbf{v}(d)\}$ $\subset R^{d-1}$ such that $\| \mathbf{v}(i)-\left.\mathbf{v}(j)\right|^{2}-|\mathbf{a}(i)-\mathbf{a}(j)|^{2} \mid<\delta$ for all $1 \leq i<j \leq d$.

Proof. Suppose wlog that $A$ is in the unit cube $[0,1]^{d-1}$. Consider the grid

$$
G(s)=\left\{0, \frac{1}{s-1}, \frac{2}{s-1}, \ldots, 1\right\}^{d-1},
$$

and let $\boldsymbol{\omega}(i) \in G(l)$ be the closest point of $G(s)$ to $\mathbf{a}(i), 1 \leq i \leq d$.

Consider also the point set $\bar{B} * \bar{B} * \cdots * \bar{B} \in R^{(d-1)(s-1)}$, where $\bar{B}=\left\{\mathbf{b}_{i} /(s-1)\right.$, $1 \leq i \leq s\}$ is defined via Lemma 4.1 .

This is something like a deformed grid. Let $\mathbf{v}(i)$ be the point in the deformed grid corresponding to $\omega(i)$.

By Theorem 2.2, the set $\{\mathbf{v}(1), \ldots, \mathbf{v}(d)\}$ is super-Ramsey.

In view of the construction, $|\mathbf{v}(i)-\mathbf{v}(j)|^{2}-|\mathbf{a}(i)-\mathbf{a}(j)|^{2} \rightarrow 0$ as $s \rightarrow \infty$. Thus, choosing $s>s(\delta)$, the corollary is proved.

\section{ALL SIMPLICES ARE SUPER-RAMSEY}

Theorem 5.1. Let $A \subset R^{d-1},|A|=d$, be an affinely independent point set. Then $A$ is super-Ramsey.

Before proving this theorem, let us recall the following characterization of Euclidean point sets.

Let $N=\left\{u_{1}, \ldots, u_{d}\right\}$ be a finite metric space with distance $d\left(u_{i}, u_{j}\right)=d_{i j}$. Let $E=\left\{e_{i j}: 1 \leq i<j \leq d\right\}$ be a set of reals. Then $E$ is said to be of negative type if

$$
\sum_{1 \leq i<j \leq d} \lambda_{i} \lambda_{j} e_{i j} \leq 0
$$

holds for all choices of $\lambda_{1}, \ldots, \lambda_{d}$ such that $\lambda_{1}+\cdots+\lambda_{d}=0$ and $\lambda_{1}^{2}+\cdots+\lambda_{d}^{2}=$ 1 .

Theorem (Schönberg [S]). A finite metric space $N$ can be embedded into Euclidean space $R^{d-1}$ if and only if the set $E=\left\{d_{i j}^{2}: 1 \leq i<j \leq d\right\}$ is of negative type. Moreover, its image is affinely independent if and only if the inequality (5.1) is strict for all choices of $\lambda_{1}, \ldots, \lambda_{d}$ (not identically zero).

Proof of Lemma 5.1. Let $\left\{e_{i j}: 1 \leq i<j \leq d\right\}$ be the squares of the distances between the points in $A$. Suppose wlog that $e_{i j} \leq 1$ for all $1 \leq i<j \leq n$. In 
view of Schönberg's theorem, there exists a positive $\gamma$ such that the left-hand side of $(5.1)$ is always less than $-\gamma$.

Define $\beta=\gamma / d^{2}$. Then the set $E^{\prime}=\left\{e_{i j}-\beta: 1 \leq i<j \leq d\right\}$ is still of negative type. Consequently, there exists $A=\{\mathbf{a}(1), \ldots, \mathbf{a}(d)\} \subset R^{d-1}$ so that

$$
|\mathbf{a}(i)-\mathbf{a}(j)|^{2}=e_{i j}-\beta, \quad 1 \leq i<j \leq d .
$$

Apply Corollary 4.2 with a small but positive $\delta=\delta(\beta)$ to obtain the set $V=\{\mathbf{v}(1), \ldots, \mathbf{v}(d)\}$.

Define $y_{i j}=e_{i j}-|\mathbf{v}(i)-\mathbf{v}(j)|^{2}$ for $1 \leq i<j \leq d$.

For $\delta$ sufficiently small, Corollary 3.2 implies that there exists a superRamsey set $B=\{\mathbf{b}(1), \ldots, \mathbf{b}(d)\} \subset R^{d-1}$ with $|\mathbf{b}(i)-\mathbf{b}(j)|^{2}=y_{i j}$.

Consider the set

$$
\{\mathbf{a}(1) * \mathbf{b}(1), \ldots, \mathbf{a}(d) * \mathbf{b}(d)\} \subset A * B .
$$

By Theorem 2.2, this set is super-Ramsey. On the other hand,

$$
|\mathbf{a}(i) * \mathbf{b}(i)-\mathbf{a}(j) * \mathbf{b}(j)|^{2}=|\mathbf{a}(i)-\mathbf{a}(j)|^{2}+|\mathbf{b}(i)-\mathbf{b}(j)|^{2}=e_{i j}
$$

for all $1 \leq i<j \leq d$, concluding the proof.

\section{RELATED AND OPEN PROBLEMS}

First of all, we made only a modest contribution towards deciding which sets are Ramsey. In particular, we do not have any example of a spherical set that is not Ramsey.

Graham [G1] introduced a related notion, stronger than being Ramsey.

Definition 6.1 [G1]. Call a set $A \subset R^{d}$ sphere Ramsey if for every $r \geq 2$ there exist an $n=n(A, r)$ and a positive real $\rho=\rho(A, r)$ such that for every $r$ coloring of the points of the sphere $S(\rho, n)=\left\{\mathbf{x} \in R^{n},|\mathbf{x}|=\rho\right\}$, there exists a monochromatic point set $A^{\prime}$ that is congruent to $A$.

Problem 6.2. Are there any Ramsey sets that are not sphere Ramsey?

For a spherical set $A$, let $\rho(A)$ denote its circumradius, i.e., the radius of the smallest sphere containing $A$.

Graham proves that all bricks are sphere Ramsey and asks whether for a brick $B$ one can choose in Definition $6.1 \rho=\rho(A)+\varepsilon$ for an arbitrary positive $\varepsilon$. Actually, much more is true.

Definition 6.3. Call a spherical set $A \subset R^{d}$ hyper-Ramsey if for all $\delta>0$ there exist positive constants $c=c(A, \delta), \varepsilon=\varepsilon(A, \delta)$, and subsets $X=X(n) \subset$ $S(\rho(A)+\delta, n)=\left\{\mathbf{x} \in R^{n}:|\mathbf{x}|=\rho(A)+\delta\right\}$ for $n>n_{0}(\delta)$ such that (i) and (ii) from Definition 2.1 hold.

Theorem 6.4. If $A \in R^{d}$ and $B \in R^{e}$ are hyper-Ramsey, then so is $A * B$.

The proof is almost exactly the same as that of Theorem 2.2 and, therefore, will be omitted.

From the results of Frankl and Wilson [FW], it follows that every two-element set is hyper-Ramsey (see [R] where this is stated explicitly). 
Corollary 6.5. All bricks (and their subsets) are hyper-Ramsey.

Our current knowledge does not exclude the possibility that all spherical sets are hyper-Ramsey, but we cannot prove this even for nondegenerate simplices.

Open Problem 6.6. Are obtuse triangles hyper-Ramsey?

\section{REFERENCES}

[E] P. Erdös, R. L. Graham, P. Montgomery, B. L. Rothschild, J. Spencer, and E. Straus, Euclidean Ramsey theorems. I, J. Combin. Theory Ser. A 14 (1973), 341-363; Euclidean Ramsey theorems. II, III, Infinite and Finite Sets (A. Hajnal, R. Rado, and V. T. Sós, eds.), North-Holland, Amsterdam, 1975, pp. 529-557 and 559-583.

[FR1] P. Frankl and V. Rödl, All triangles are Ramsey, Trans. Amer. Math. Soc. 297 (1986), 777-779.

[FR2] _ Forbidden intersections, Trans. Amer. Math. Soc. 300 (1987), 259-286.

[FRo] P. Frankl and I. G. Rosenberg, A finite set intersection theorem, European J. Combin. 2 (1981), 127-129.

[FW] P. Frankl and R. M. Wilson, Intersection theorems with geometric consequences, Combinatorica 1 (1981), 357-368.

[G1] R. L. Graham, Old and new Euclidean Ramsey theorems, discrete geometry and convexity, Ann. New York Acad. Sci. 440 (1985), 20-30.

[G2] _ Rudiments of Ramsey theory, CBMS Regional Conf. Ser. in Math., no. 45, Amer. Math. Soc., Providence, RI, 1981.

[R] V. Rödl, On a problem in combinatorial geometry, Discrete Math. 45 (1983), 129-131.

[S] I. J. Schönberg, Metric spaces and positive definite functions, Trans. Amer. Math. Soc. 44 (1938), 522-536.

ABSTRACT. Given the vertex set $A$ of a nondegenerate simplex in $R^{d}$, it is shown that for some positive $\varepsilon=\varepsilon(A)$ and every partition of $R^{n}$ into fewer than $(1+\varepsilon)^{n}$ parts, one of the parts must contain a set congruent to $A$. This solves a fifteen-year-old problem of Erdös et al. [E].

Centre National de la Recherche Scientifique, 15 Quai Anatole France, Paris, France 75007

Department of Mathematics, Emory University, Atlanta, Georgia 30322 